Revista Arbitrada Interdisciplinaria KOINONIA

Año V. Vol V. Nº. Enero - Junio 2020

Hecho el depósito de Ley: FA2016000010 ISSN: 2542-3088

FUNDACIÓN KOINONIA (F.K). Santa Ana de Coro. Venezuela.

Luis Urrego

http://dx.doi.org/10.35381/r.k.v5i9.556

\title{
Liderazgo Estratégico: Perspectiva clave hacia la responsabilidad social en Instituciones de Educación Superior
}

\section{Strategic Leadership: Key perspective towards social responsibility in Higher Education Institutions}

\author{
Luis Urrego \\ lurrego2@unisimonbolivar.edu.co \\ Universidad Simón Bolívar \\ Colombia \\ https://orci.org/0000-0002-9210-4338
}

Recibido: 1 de julio de 2019

Revisado: 30 de julio de 2019

Aprobado: 20 de agosto de 2019

Publicado: 20 de enero de 2020

\begin{abstract}
RESUMEN
El alcance general de este estudio es comprender el liderazgo estratégico como perspectiva clave hacia la responsabilidad social en instituciones de educación superior. Sustentado en autores como Hax y Majluf, (2009), Etkin (2011), entre otros, además aportes que hagan los informantes, así como la opinión del mismo investigador. Bajo esta perspectiva, se opta por la etnografía como método, orientado desde la corriente de pensamiento interaccionismo simbólico con enfoque introspectivo vivencial. La información se obtuvo mediante la observación y entrevistas semiestructuradas. Los resultados indicaron que la evolución del contexto presiona a las organizaciones a la formulación de estrategias con la finalidad de minimizar los efectos negativos de la competencia sobre su desempeño y al mismo tiempo aprovechar oportunidades. Este dinamismo en el que se encuentran las organizaciones promueve cambios que alientan a la renovación constante de los objetivos, así como también modifica las preferencias de la gente a quien dirigen sus esfuerzos.
\end{abstract}

Descriptores: Liderazgo; responsabilidad social; responsabilidad social; desarrollo económico y social. (Palabras tomadas del Tesauro UNESCO).

\begin{abstract}
The general scope of this study is to understand strategic leadership as a key perspective towards social responsibility in higher education institutions. The research is sustained in authors such as (Hax and Majluf, (2009), Etkin (2011), among others, in addition to the contributions made by the informants, and the opinion of the same researcher.). From this perspective, ethnography is chosen as
\end{abstract}




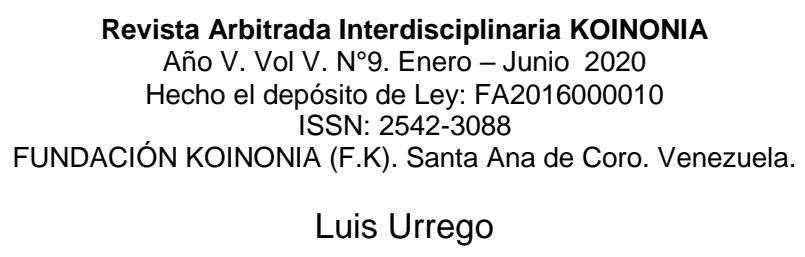

a method, oriented from the current of thought: symbolic interactionism with an experiential introspective approach. Based on this, information was obtained through observation and semi-structured interviews. The results indicated that the evolution of the context puts pressure on organizations to formulate strategies in order to minimize the negative effects of competition on their performance and, at the same time, take advantage of opportunities. This dynamism in which organizations find themselves promotes changes that encourage the constant renewal of objectives, as well as the modification of people's preferences to whom they direct their efforts.

Descriptors: Leadership; social responsability; Social responsibility; Economic and social development. (Words taken from the UNESCO Thesaurus).

\section{INTRODUCCIÓN}

En el mundo contemporáneo, en varios ámbitos de los diferentes sectores ha brotado la necesidad de descubrir nuevas formas de afrontar retos, que se les aplican a los individuos, organizaciones y estados, donde el liderazgo se esboza como la estrategia que permite a los ejecutivos consumar con las metas planteadas. Así, la gestión pragmática moderna debe estar bien idónea con conocimientos administrativos, para responder a los desiguales escenarios, donde las instituciones están comprometidas y deben adecuarse a los efectos generados por la globalización.

En el mismo sentido, es significativo aludir que existen incontables esfuerzos realizados por los conductistas por introducir las diferentes teorías de liderazgo, haciendo hincapié en organizarlas. Además, las teorías contemporáneas destacan como última corriente, el estudio de la teoría del liderazgo estratégico, la cual se caracteriza por tener un carácter estratégico, situacional y sistemático.

Habitualmente, se especula al líder como un individuo aislado que conduce aspiraciones, metas de un grupo y como si fuera un personaje solitario, tomando las riendas logrando victorias demandadas para triunfar. Nada más lejos de la concepción esencial del liderazgo, esto es un fenómeno dual, pues no existe líder sin seguidores. El líder nace de una necesidad de todo grupo humano quien consciente 0 inconscientemente sabe que requiere de orden, dirección, organización e integración.

Es por ello que pueda decirse que hoy, en este mundo complejo, el binomio 
líder/seguidores cobra mayor importancia en las organizaciones modernas, en las que los seguidores tienden a influir más en las decisiones, y a participar en los resultados de las instituciones. Actualmente se tienen millones de trabajadores que forman parte de círculos de calidad, en los cuales, entre todos sus miembros, analizan los problemas, miden efectos, escogen soluciones y las aplican.

A este respecto, se está viviendo una renovación, un fortalecimiento de la interacción de los líderes con sus seguidores, en un modelo más participativo y corresponsable de ambas partes. Sin embargo, se sabe que especialmente en las organizaciones modernas, se requiere que no sólo exista un buen liderazgo en la cabeza, sino en todos los niveles o áreas de la institución existan verdaderos jefes, guiando los esfuerzos de sus equipos para formar la red o estructura de liderazgo de toda la organización.

En tal sentido, en la mayoría de los casos, quizás el éxito o fracaso de la empresa, se le atribuiría a la forma cómo han sido gerenciadas; asimismo, se debe tener en cuenta tanto los modelos organizacionales, como las acciones de la administración estratégica basada en un conjunto de pautas, reglas, normas y principios que guíen la acción de los individuos para el logro de metas comunes. No obstante, cabe destacar, que sin una estrategia efectiva del liderazgo, la institución sería capaz de influenciar el ambiente externo para su beneficio, razón por lo cual, el líder estratégico tiene la responsabilidad de convertirse en un experto de la información y de la influencia.

Por lo tanto, es importante mencionar lo esencial de saber qué está pasando dentro y fuera de la organización, en su ambiente interno y externo; con miras a conocer las oportunidades e influenciar tales eventos para la consecución de los objetivos de la organización; es decir, el líder estratégico debe poseer una red de asesores o colaboradores influyentes en ambos ambientes, es decir, estar en contacto directo con todas aquellas personas o líderes de cualquier ideología que tengan la capacidad de influir directa e indirectamente en el gobierno o la opinión pública.

Por ello entonces podría decirse que toda empresa competitiva requiere de líderes estrategas, proactivos, capaces de enfrentar retos o cambios garantizando la permanencia en los mercados, especialmente en empresas con pocos recursos 
materiales, técnicos o que han descuidado significativamente el humano. Al igual que los entrenadores de los equipos deportivos, trabajan directamente en crear una mente positiva de triunfo con expectativas alentadoras del futuro. Así, la percepción positiva del mundo y la relación del líder sobre sus seguidores, influye necesariamente sobre los logros.

Es decir, las empresas ya no son sólo fuente de trabajo y productoras de dinero, sino que también son vista como centros de aporte para el desarrollo de las comunidades en sus distintos ámbitos, como el educativo, cultural, social, deportivo, entre otros; acción de desarrollo y aporte a la sociedad que es entendida como responsabilidad social empresarial.

\section{DESARROLLO TEÓRICO}

\section{Liderazgo Estratégico}

Por décadas el ser humano se ha interesado por la definición y composición del liderazgo, por tanto los individuos logran altos niveles de productividad y eficiencia, es a partir de 1950 donde se investiga sobre el perfil de los líderes, su influencia en los medios y sus habilidades, inclusive como una persona con rasgos distintivos de personalidad hace que sobresalga como un líder. Por otra parte, históricamente, el ser humano se siente proclive a esgrimir sus buenos roles de liderazgo para cultivar sus capacidades para motivar e influir.

Los líderes Estratégicos deben tomar decisiones importantes para lograr el éxito dentro de las organizaciones, donde los directores o los niveles altos siempre observan muy de cerca el entorno externo e interno, en la búsqueda contante para descubrir oportunidades del mercado, medir las capacidades, lograr reconocer las amenazas, desarrollar una visión de futuro, anticiparse en los cambios que pueden experimentar las empresas.

En este orden de ideas, se puede citar a Griffin (2011) quien define el liderazgo estratégico como la capacidad de entender las diferentes complejidades que experimenta la organización y su entorno. De modo que, es un nuevo concepto que lo relaciona con la alta gerencia, para ser efectivo en este rol, el líder debe conocer de la organización su historia, las fortalezas, su cultura, así como sus debilidades, además como la empresa está alineada con el entorno, las 
tendencias para mejorar la alineación actual y futura.

Por su parte, Blanchard (2007) lo define como el aspecto del liderazgo que está asociado con la orientación, visión clara, cultura, servicio, además, asegurando que todos dentro de la organización vayan en la misma dirección, alineando el conjunto de valores con la visión, así como con iniciativas de fácil ejecución. Por consiguiente, los valores y la visión son perdurables y los imperativos estratégicos son a corto plazo con duración que van de uno a dos meses o uno a dos años.

Mientras Etkin (2006), define liderazgo estratégico como la capacidad de moverse atendiendo varios frentes a la vez, que no son complementarios, cuando se está en presencia de demandas y presiones contradictorias. En consecuencia, en el contexto cambiante este concepto no se circunscribe sólo a alcanzar que las cosas sean ejecutadas por las personas, en las situaciones de incertidumbre, cambios, no es suficiente la habilidad o carisma para comunicarse, de manera que, sobre un entorno totalmente competitivo, los líderes deben tener la capacidad de movilizar al talento humano para compartir los proyectos, esto se consigue con una visión de futuro, revisión de los procesos y una actitud abierta.

Al contrastar los postulados de los diferentes autores, se percibe convergencia en plantear que el liderazgo estratégico es un factor determinante en el desarrollo de la organización, impactando a su vez en el direccionamiento estratégico, agregando dinámica y destrezas a los procesos, buscando innovación que involucra la transformación hacia lo nuevo.

En términos generales, se define el liderazgo estratégico como la capacidad de anticiparse, dar poder a las personas para generar transformación o cambio estratégico esperado dentro del negocio de tarjeta de crédito, a través de la motivación y la buena dirección, impactando sobre la fortaleza de las instituciones de Educación Superior, con lo cual se genera dinámica en los procesos que dan paso a la innovación y la transparencia hacia lo nuevo.

\section{Características del liderazgo estratégico}

Las características más relevantes del liderazgo estratégico son los roles del líder estratégico, permiten puntualizar las actuaciones del director en cuanto al desempeño que se espera de él como principal accionado de la estrategia 


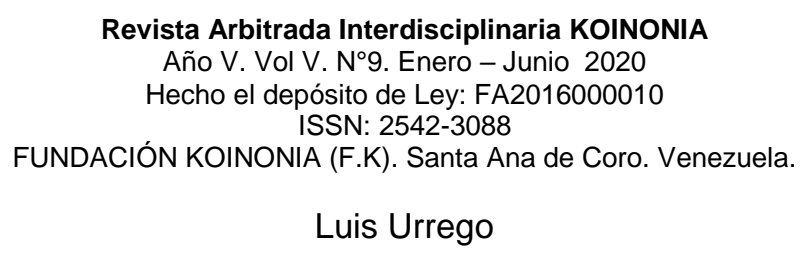

institucional. En este sentido, Flores (2009 p.83), señala que los roles del director de organizaciones educativas, "...permiten analizar y redimensionar el hecho educativo como un proceso interactivo constructivo, en el cual la relación directivo-docente-alumno-comunidad, estrategia y evaluación se integran en un solo objetivo".

De acuerdo con este planteamiento, los roles del líder estratégico evidencian sus actuaciones dentro de las líneas que orientan la labor educativa, de acuerdo al propósito de crear condiciones favorables al logro de las metas establecidas. Tomando en cuenta, el planteamiento anterior, los roles del líder estratégico permiten caracterizar sus actuaciones en atención al desempeño que se espera de él como profesional de la educación y agente de cambio social.

En efecto, estos roles para Borjas (2009 p.56) "se asocian a las distintas funciones que desempeña el director como gerente educativo e impulsador de alternativas para la solución de los diferentes problemas afrontados, orientando eficazmente la interacción entre las experiencias y necesidades detectadas"; esto le permite atender adecuadamente la diversidad manifiesta en la compleja dinámica de los procesos educativos, la cual guía con voluntad para alcanzar la transformación deseada en las organizaciones.

En este sentido, los roles del líder estratégico se asocian a indicadores que modifican favorable o desfavorablemente la gestión que ejecuta tanto en el ámbito interno de la institución de educación superior, como en el contexto externo o comunitario; de allí, que Rojas, C. (2009), destaque características que deben prevalecer en el rector como gerente educativo, las cuales configuran la orientación que imprimen a su desempeño, entre ellas: Creativo, honesto, optimista, perseverante, reflexivo y crítico.

Dentro de este marco, se percibe que el rector al asumir los roles del líder estratégico requiere desarrollar actitudes y valores personales en atención a sus responsabilidades; es decir, adquirir habilidades que le permitan interactuar de manera eficaz con las distintas circunstancias inherentes a la gerencia educativa. Es así, como el rector asigna a su gestión educativa, las concepciones que tiene acerca del mundo, la vida y el conocimiento, a través de los roles que caracterizan cada una de las funciones a su desempeño. 
Al respecto, Goodstein (2009), considera que "la implementación de acciones bajo el enfoque de la gerencia estratégica, exige la presencia de directivos comprometidos con el desarrollo y alcance de las metas organizacionales" (p.83), para lo cual es propicio configurar un desempeño basado en roles ejecutados dentro de la organización.

Continuando con los autores antes citados, los roles del líder estratégico se constituyen alrededor de tres aspectos centrales los cuales son: establecer el escenario para la planeación estratégica, lo cual implica ayudar a que los participantes comprendan el proceso de dirección y la forma como opera; el establecimiento de direcciones estratégicas, implicando el diseño de la estrategia, la auditoria de desempeño y el análisis de dificultades; la implementación, el cual involucra la integración de los planes de acción, la planeación de contingencias y la puesta en marcha.

Cabe destacar, que la ejecución de estos roles, requiere ser orientada desde una perspectiva integral, a fin de abarcar todas las funciones inherentes a la gerencia educativa. Desde esta perspectiva, los roles del líder estratégico en las instituciones de educación superior, delinean el desempeño que se espera de él; es decir, concretizan las competencias que debe poseer en su acción dentro y fuera de la institución educativa.

Actualmente, la función del rector es traducida como el impulsador de procesos donde todos los actores educativos participen activamente en la dinámica escolar; además, se le atribuye la responsabilidad de lograr los objetivos de la educación para lo cual requiere desplegar sus conocimientos y habilidades a fin de contribuir al logro de las metas planteadas.

En esta línea de pensamiento, Pérez (2009), enfatiza que los roles del líder estratégico, asociados al desempeño de los rectores de las instituciones de educación superior, concretizan sus actuaciones dentro de las líneas que orientan la labor educativa, de acuerdo al propósito de crear condiciones para el encuentro entre las transformaciones pautadas y la concreción de ellas a través de la incorporación continua de acciones innovadoras.

En resumen, el rector de las instituciones de educación superior objeto de estudio, al asumir la gestión educativa desde la perspectiva del liderazgo estratégico, 
organiza y orienta cuidadosamente, el despliegue de múltiples acciones a fin de dar respuesta a los diferentes procesos implícitos en la dinámica escolar; lo cual es posible abordarlo por medio de los roles que como líder estratégico le caracterizan.

\section{Responsabilidad Social}

En relación a la responsabilidad social Fernández, (2009) manifiesta que es el compromiso que tienen todos los ciudadanos las instituciones públicas y privadas organizacionales sociales en general para contribuir al aumento del bienestar la sociedad local y global. De la misma manera ha de ser atendida como una filosofía, actitud que adopta la empresa hacia los negocios reflejando en la incorporación voluntaria su gestión de las preocupaciones, expectativas de distintos grupos de interés con una visión a largo plazo.

Así mismo, una empresa socialmente responsable busca el punto óptimo de cada momento, entre la rentabilidad económica, mejora del bienestar social de su comunidad y preservación del medio ambiente. La responsabilidad social corporativa, amplía el ámbito de la responsabilidad social de la empresa para incorporar a las agencias gubernamentales y a otras organizaciones, que tengan claro el interés en mostrar como realizan su trabajo.

Según Robbins, (2009), explica que para entender mejor la responsabilidad social; se debe comparar dos conceptos afines. La obligación social es el deber de una empresa, cumplir con su responsabilidad económica y legal. La organización hace únicamente lo que está obligado hacer y expresa una postura tradicional sobre la responsabilidad social. En contraste con la obligación social la responsabilidad social, la sensibilidad social da un paso al cumplimiento con las normas básicas y legales.

De igual manera, define la responsabilidad social como el deber de las empresas, apartes de los requisitos legales y económicos, de perseguir metas de largo plazo para bien de la sociedad. La responsabilidad social añade el imperativo moral de hacer aquello que mejora la sociedad de no hacer lo determinado. Esta requiere que las empresas determinen lo bueno, lo malo al tomar, decisiones éticas y entregarse a las actividades éticas. Una organización con responsabilidad social 


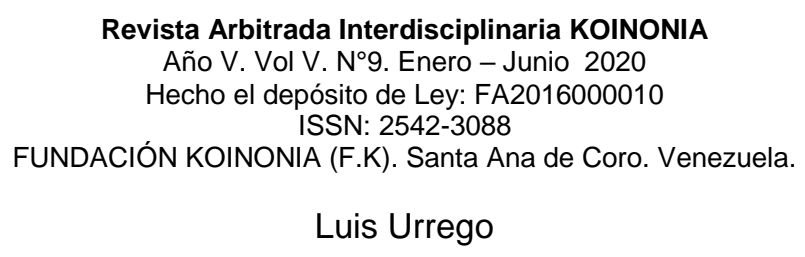

hace lo correcto por qué le parece que está obligada a conducirse de tal manera. En este orden de ideas Guédez (2008) sostiene la incuestionable importancia de la responsabilidad social empresarial ha despertado una creciente curiosidad, así como una amplia cantidad de especulaciones e hipótesis. Para algunos es más de lo mismo, otros dicen que es una moda pasajera, hay quienes sostienen que es una imposición que viene desde afuera, también existen los que interpretan como un enclave organizacional e, incluso, aquellos que aseveran que ella es un artificio para nuevos negocios y, desde luego están los que preconizan que es un modelo de gestión.

De esta manera, define la responsabilidad social como una visión de negocios que integra a la gestión de la empresa, el respeto por: los valores y principios éticos, los trabajadores, la comunidad y el medio ambiente. Esto quiere decir que es un interés propio e inteligente de la empresa que produce beneficios a la comunidad y a la propia empresa.

Asimismo, Reyno (2007) en su artículo sostiene que la responsabilidad social empresarial renueva la concepción de la empresa, otorgando a ésta una dimensión amplia e integradora, que va más allá de la mera cuestión económica en la que se incorpora perfectamente la triple faceta de la sostenibilidad: económica, social y medioambiental. El desarrollo sostenible se sitúa como fin a alcanzar por medio de la adecuada implantación de un modelo de empresa socialmente responsable, en el que los distintos grupos de interés, stakeholders, son el centro de atención esencial para la gestión.

Por tanto entonces, es importante señalar que la responsabilidad social solamente se fortalece para la realización de trabajos sociales en la comunidad, sino es así entonces se trata más bien de una estrategia de las instituciones, que junto a su gestión de negocios y reflejando sus valores, debe ser tratada como un caso de negocios más.

\section{Estrategias de la Responsabilidad Social}

Toda organización debe centrarse en una organización estratégica para poder garantizar la perdurabilidad en el tiempo. El objetivo fundamental de las empresas se puede expresar como la consecución de una rentabilidad superior al coste de 


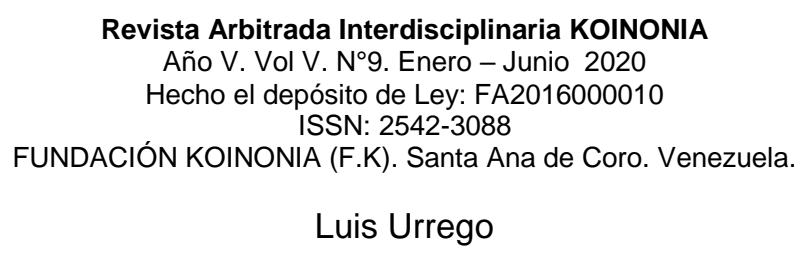

oportunidad del capital invertido y a ellos contribuirán los dos niveles básicos de las estrategias de la responsabilidad social.

De allí entonces, que la responsabilidad social es una tarea que requiere del esfuerzo transversal de todos los colaboradores de la empresa. Como se trata de una manera innovadora de hacer el negocio, es necesario lograr el compromiso de la alta dirección para impulsar el cambio cultural necesario, y el de cada uno de los trabajadores, para transferir esa visión a su labor del día a día.

En consecuencia, dependiendo del tipo de negocio, y de los riesgos y oportunidades detectados, cada empresa desarrollará las políticas, cambios e iniciativas que mejor respondan a sus necesidades de adaptación a las nuevas exigencias sociales y medioambientales del entorno en el que se desenvuelve. De esta manera, la organización aumentará su competitividad y posibilidades de subsistir en el largo plazo.

Al respecto Guédez (2008), manifiesta que la responsabilidad social es estratégica para las empresas, porque además de generar resultados, impactos y retornos, es también objeto de diseños que alinean con los negocios sustantivos de las organizaciones. Ciertamente la responsabilidad social no es el negocio, pero es parte del negocio de manera análoga al hidrogeno que no es agua pero es parte del agua.

En opinión de Fernández (2008), la visión estratégica de la empresa está basada en dos niveles básicos de estrategias, estrategias corporativas y estrategias de negocio o competitiva, condicionan decisivamente la consecución de una rentabilidad superior al coste de oportunidad del capital invertido. En primer lugar, el hecho de estar en un determinado sector y mercado con unas o más condiciones más o menos favorables influirá en la rentabilidad de la empresa.

Del mismo modo, Morros (2006), considera que la estrategia proviene de la acción pública por tanto entonces puede notarse como coinciden en expresar que las estrategias deben considerarse en un concepto multidimensional que abarca a toda organización, otorgándole sentido del sistema abierto, perfectamente delimitado con su entorno, que interacciona, en los diferentes tipos de procesos y opresiones, información y decisiones son reconocibles y orientados. 


\section{Luis Urrego}

\section{METODOLOGÍA}

Desde el paradigma, del pospositivismo se menciona lo estudiado por Blasco y Pérez, (2007), quien plantea que este se refiere al conjunto de suposiciones interrelacionadas respecto al campo social que proporciona un marco filosófico del estudio organizado de este mundo, en otras palabras, pasa hacer una visión del mundo, desde la perspectiva general, es un modo de desmenuzar la complejidad del mundo real.

Bajo esta visión, el paradigma cualitativo nace de la necesidad de ampliar la comprensión del entorno, con una cara opuesta al positivismo y sus ciencias exactas, por lo que, se intenta un abordaje más amplio en cuanto al conocimiento de una sociedad compleja, con individuos que piensan y actúan de una manera distinta, dando paso a una amplia gama de oportunidades para interpretar el mundo.

Desde este enfoque cualitativo, Grove y Burn (2014), consideran que la investigación es una aproximación sistemática y subjetiva que va permitir describir las experiencias de vida y darles un significado, centrado en la comprensión de la globalidad, por ello, explora la profundidad, riqueza y complejidad inherentes a un fenómeno.

En este sentido, el investigador se adentra en el escenario para observar e interactuar con las personas, desde una perspectiva holística; considerando además que los escenarios o grupos no son reducidos ni variables, sino más bien considerados como un todo, por ello, el investigador cualitativo estudia a los sujetos en su contexto pasado, evaluando las situaciones en las que se encuentre actualmente.

Desde esta orientación, el método que se ha seleccionado para la investigación es la etnografía, debido a que esta se circunscribe a los desarrollos conceptuales que ha tenido en la tradición antropológica, la misma goza de reconocimiento en materia de investigación cualitativa. En otras palabras, la etnografía, se refiere al método, al enfoque, pero también al capítulo, en el que se hace la descripción escrita de las formas de interacción de una comunidad.

Asimismo, se elige la etnografía, porque permite reunir elementos suficientes, en términos delo cualitativo, el interaccionismo simbólico y el enfoque introspectivo 
vivencial, tanto como las técnicas que se eligen para esta investigación. Entonces, la etnografía es pospositivista por cuanto se erigió como alternativa ante los métodos cuantitativos, de igual forma, su carácter cualitativo se hace evidente porque uno de los puntos de mayor interés es describir cómo las personas otorgan sentido a las cosas de su realidad cotidiana.

Hoy día, la etnografía estudia las comunidades que viven al ritmo actual que impone la globalización y las nuevas tecnologías, de forma, que ha cambiado su concepción, ya que no solo se trata de estudiar sociedades aisladas, sino de describir las acciones de un grupo humano en específico, considerando el marco de un sistema social, en el cual, existen situaciones que están influenciadas por hechos globales.

Bajo la perspectiva de este método, el investigador, ocupa un lugar central como instrumento del conocimiento, desde la experiencia investigativa o vivencial, se convierte en un sujeto cognoscente que recorre el arduo camino delo desconocido al reconocimiento, esto es, pasar hacer una persona dispuesta a conocer, a dejar atrás la ignorancia ya que, como intelectual o académico, puede codificar de tal manera que sea comprensible para todos.

Siguiendo en la misma vertiente, del enfoque introspectivo vivencial, para Guber (2011) este puede ser un obstáculo del conocimiento o su facilitador, tomando en cuenta lo que tiene que ver con articular la teoría y lo empírico. Sin embargo, las ciencias sociales, desde hace tiempo reconoce la mediación del investigador en el proceso de construcción del conocimiento, por lo que, según el investigador de este estudio, el éxito etnógrafo dependerá del proceso sistemático, tanto como de su nivel de consciencia frente a sus acciones, además de la perspectiva epistemológica con la que conciba sus prácticas investigativas frente a la comprensión e interpretación de lo que ha definido como su objeto de estudio.

Por su parte, Hammersley y Atkinson (1994) cuando se refieren a la etnografía como método, evidenciando su carácter introspectivo vivencial, ya que para ellos se enmarca en un método de investigación social, aun cuando sea poco común, debido a que trabaja con una amplia gama de fuentes de información.

Por otro lado, el análisis de los datos se manejó bajo la técnica de codificación y triangulación de datos, que según Gurdián-Fernández (2007) permite reconocer 
categorías en los datos; que no otra cosa que la concesión de un código (símbolo o abreviatura) utilizado para la codificación e identificación de palabras, por lo cual, este proceso se ejecuta con una exploración de las semejanzas y las discrepancias existentes entre los temas clave que serán conseguidos en la rotulación de las entrevistas.

Luego se procede a la triangulación, la cual consiste en realizar un pre análisis de las entrevistas, para contrastar los datos, consiguiendo con ello las unidades de análisis. De acuerdo a Martínez (2010) esta consiste en manejar una diversidad de datos para efectuar un estudio, derivados de distintas fuentes de información con la intención de contrastar la información obtenida, la misma, se desarrolló en la descripción de las categorías interpretativas, en las que se confrontarán las informaciones de los informantes clave y las acciones desarrolladas por ellos en las entrevistas.

\section{REFLEXIONES FINALES}

El estudio y análisis del presente artículo propone hacer énfasis en la importancia que tiene el liderazgo estratégico y la responsabilidad social. Lo cual permite afirmar que las nuevas generaciones en deberán liderar procesos impostergables con miras a conformar una sociedad justa y de mayor bienestar económico y social orientado con base en valores, principios democráticos, de justicia social, igualdad y solidaridad.

Uno de los mayores aportes que hace la teoría del capital social a las ciencias administrativas y del liderazgo lo constituye la consolidación de la confianza, la responsabilidad social y ética, el compromiso social y el empoderamiento, como herramientas que garantizan el éxito organizacional, ante esta nueva realidad los líderes dejan de ver la realidad como algo externo y controlable desde un punto de vista objetivo y desprendido de sus valores y sentimientos y "comienza la aceptación del otro como legítimo otro en la convivencia y en la diversidad".

Asimismo, se puede decir que la conceptualización del líder social es bastante compleja, ya que para las comunidades, un líder social no sólo es quien se preocupa de solucionar una problemática, sino que también es la persona capaz de darse cuenta que dentro de sus sistemas comunitarios ya sea amigos, padres, 
vecinos, compañeros, existen necesidades y el actúa como mediador de conflictos,

adentrándose en su entorno para realizar acciones no reconocidas.

En este orden de ideas, se hace necesario que nuestros líderes sean capaces de generar un proceso formativo colectivo, donde destaquen los valores propios y al mismo tiempo se cree conciencia sobre la necesidad de que la población participe activamente en la identificación de los problemas comunes y en la organización para resolverlos, y promueva cambios para acelerar los procesos productivos generadores de riqueza que se requieren para hacer frente a las nuevas realidades globales.

En este sentido, el liderazgo estratégico y social se convierte en una mezcla de diferentes habilidades de liderazgo, en el cual uno de los focos estratégicos es crear y sostener un clima organizacional donde los seguidores tengan éxito y la gerencia participe en la construcción y sostenimiento de relaciones en las comunidades, generando una interacción sinérgica de tipo democrático entre estado y comunidad. Esta relación sinérgica donde cada una de las partes pone lo mejor de sí respetando al otro, fomenta el capital social en la medida que favorece la confianza, las relaciones de colaboración y la capacidad para trabajar en torno a objetivos e intereses sentidos, logrando un desarrollo sostenible.

\section{FINANCIAMIENTO}

No monetario.

\section{AGRADECIMIENTO}

A los docentes de las diversas instituciones de educación superior que apoyaron el proceso investigativo. 


\section{REFERENCIAS CONSULTADAS}

Blanchard, D (2007) Clima Organizacional y Desempeño Gerencial del Personal Directivo en La E.B. "Dr. Francisco Espejo". [Organizational Climate and Management Performance of Management Personnel at "Dr. Francisco Espejo" School.]. Universidad de Carabobo Facultad de Ciencias de La Educación.

Blasco, J y Pérez, J (2007). Metodologías de investigación en las ciencias de la actividad física y el deporte. [Research methodologies in the sciences of physical activity and sport.]. España: Editorial Club Universitario. ECU.

Borjas, G(2009) La Gestión Educativa al servicio de la Innovación. [Educational Management at the service of Innovation.]. Ediciones Fe y Alegría. Caracas.

Etkin, J (2006) Liderazgo estratégico. [Strategic leadership.]. Leadership-Agenda.

Fernández, E (2009) Administración de Empresas. Un Enfoque Interdisciplinar. [Business Administration. An Interdisciplinary Approach.]. Editorial Paraninfo. España.

Flores, L (2009) Liderazgo, inversión y Toma de decisiones en la organización. Universidad de Concepción.

Goodstein, L (2009) Planeación Estratégica Aplicada. [Applied Strategic Planning]. Reimpresión. Mc Graw Hill Colombia.

Griffin, R (2011) Administración. [Administration]. Décima Edición.

Groven S y Burns N, (2004) Introducción a la investigación cualitativa. En: Investigación en Enfermería ( $3^{a} \mathrm{Ed}$.). .). . [Introduction to qualitative research. In: Nursing Research (3rd Ed.).].Elsevier España S.A; (11) 385430.

Guber, R (2011), Método, campo y reflexividad. Enciclopedia latinoamericana de sociocultura y comunicación. [Method, field and reflexivity. Latin American Encyclopedia of Socioculture and Communication.]. Grupo Editorial, Norma.

Guédez, V. 2008) Ser Confiable. Responsabilidad Social y Reputación Empresarial. [Be reliable. Social Responsibility and Business Reputation]. Editorial Planeta Venezolana S.A, Caracas, Venezuela. 
Gurdían-Fernández A. (2007). El Paradigma Cualitativo en la Investigación SocioEducativa Alicia Gurdián-Fernández Coordinación Educativa y Cultural Centroamericana (CECC) Agencia Española de Cooperación Internacional (AECl) San José, Costa Rica. [Be reliable. Social Responsibility and Business Reputation]. Colección: Investigación y Desarrollo Educativo Regional (IDER).

Hammersley, M. y Atkinson, P. (1994). Etnografía. Métodos de investigación. [Ethnography. Research Methods]. Barcelona: Paidós.

Martínez, M. (2010). Evaluación cualitativa de programas. [Qualitative evaluation of programs]. Psicoprisma. № 1. AVEPSO. Caracas.

Morros (2006), El Control De Gestión Estratégica En Las Organizaciones. [Qualitative evaluation of programs]. CICAG, Volumen 6 - Edición 1 - Año 2009.

Pérez, J (2009) Administración del Comportamiento Organizacional. Liderazgo Situacional. [Organizational Behavior Management. Situational leadership]. Editorial Prentice. Séptima Edición. México.

Reyno, M (2007) La Responsabilidad Social Empresarial como ventaja competitiva. [Corporate Social Responsibility as a competitive advantage]. Septiembre 2007.

Robbins, S (2009) Administración. [Administration]. Editorial Pearson Educación.

Rojas, C. (2009) Recursos Humanos y Administración. [Human Resources and Administration]. Editorial Trillas. México. 